\title{
Postresection Period-Specific Hazard of Recurrence as a Framework for Surveillance Strategy in Patients with Hepatocellular Carcinoma: A Multicenter Outcome Study
}

\author{
Ha II Kim ${ }^{a}$ Jihyun An ${ }^{b}$ Ji Yoon Kim ${ }^{c}$ Hyun Phil Shin ${ }^{a}$ Seo Young Park \\ Gi-Won Song ${ }^{e, f}$ Han Chu Lee ${ }^{c, e}$ Ju Hyun Shim ${ }^{c, e}$
}

${ }^{a}$ Gastroenterology, Kyung Hee University Hospital at Gangdong, Seoul, Republic of Korea; ${ }^{b}$ Gastroenterology and Hepatology, Hanyang University College of Medicine, Guri, Republic of Korea; ' $\mathrm{C}$ Gastroenterology, Asan Medical Center, University of Ulsan College of Medicine, Seoul, Republic of Korea; ${ }^{\mathrm{d}}$ Statistics and Data Science, Korea National Open University, Seoul, Republic of Korea; ${ }^{\mathrm{e}}$ Asan Liver Center, Asan Medical Center, University of

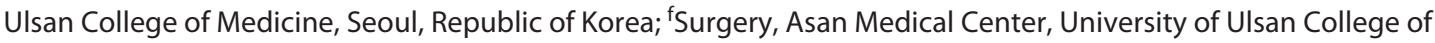
Medicine, Seoul, Republic of Korea

\section{Keywords}

Liver cancer · Resection · Outcome · Surveillance

\begin{abstract}
Introduction: In spite of the high frequency of recurrence of hepatocellular carcinoma (HCC) after resection, little evidence exists to directly help to plan a reasonable schedule for the frequency and intensity of postoperative surveillance for recurrence. Methods: 1,918 consecutive patients with Child-Turcott-Pugh class A who had T1- or T2-staged HCCs detected by active surveillance and underwent curative resection for their tumors at 3 teaching hospitals in Korea, followed by recurrence screening at 6-monthly or shorter intervals. To set an evidence-based timetable for postoperative surveillance, we investigated the annual hazard rate of recurrence through postoperative year 10 in patients undergoing hepatectomy for HCC, and the clinical and morphological phenotypes associated with early versus late recurrence. $\boldsymbol{R} \boldsymbol{e}$ sults: The estimated hazard rate for recurrence peaked dur-
\end{abstract}

karger@karger.com www.karger.com/lic

Karger $\stackrel{\text { ' }}{5}$

GOPEN ACCESS
(C) 2021 The Author(s)

Published by S. Karger AG, Basel

This is an Open Access article licensed under the Creative Commons Attribution-NonCommercial-4.0 International License (CC BY-NC) (http://www.karger.com/Services/OpenAccessLicense), applicable to the online version of the article only. Usage and distribution for commercial purposes requires written permission. ing year 0-1 (21.7\%), with a subsequent gradual decrease through 5 years, followed by stabilization at $<7.0 \%$ until year 10 , except in the case of cirrhotics, who had a rate of $10.5 \%$ during year $4-5$. Multivariate time-to-recurrence analysis by recurrence period revealed that serum alpha-fetoprotein $\geq 200 \mathrm{ng} / \mathrm{mL}$, larger size of tumor, tumor multiplicity, microvascular invasion, capsular invasion, and higher METAVIR fibrosis stage were significantly related to disease recurrence within 5 years after resection, while cirrhosis (METAVIR F4) alone was related to disease recurrence beyond 5 years ( $P S$ $<0.05$ ). Post-relapse overall survival was better in the latter group ( $p=0.033$ ). Conclusions: Our chronological and morphological insights into recurrence after resection of primary HCCs may help implement an optimal intensity of surveillance for recurrence.

(c) 2021 The Author(s).

Published by S. Karger AG, Basel

Ha Il Kim and Jihyun An contributed equally to this work.
Correspondence to:

Ju Hyun Shim, s5854@amc.seoul.kr 


\section{Introduction}

Hepatic resection is one of the potentially curative therapies in patients with hepatocellular carcinoma (HCC) and preserved hepatic function. However, given a cumulative HCC recurrence after surgery of up to $70 \%$ at 5 years, regular surveillance in the postoperative phase is crucial for early detection of treatable recurrence, which favors long-term survival $[1,2]$.

The timing of recurrence is generally associated with biological factors indicative of tumor aggressiveness, with earlier episodes having a more negative prognosis [3-7]. Genetic and molecular studies focused specifically on the clonal origins of recurrent HCCs have identified differences between the characteristics of earlier and later recurrences $[8,9]$.

Some previous postsurgical investigations have reported a second peak in the incidence of recurrence at around 4 years after HCC resection that could be mainly attributable to de novo primary tumors arising from livers with more cancer-prone backgrounds $[10,11]$. However, although the most recent global guidelines only recommend follow-up (at 3-4 month intervals) for the first year after resection, there is no formal consensus on the approach to regular postresection surveillance for recurrent HCC in subsequent years [2].

Most importantly, statistical risk analysis of tumor recurrence should be a precondition for efficient scheduling of surveillance after radical treatment [12]. The hazard function rather than the cumulative probability curve would seem to be optimal for the relevant analysis, as it provides an information-rich view of the recurrence pattern over time. Accordingly, to set an evidence-based timetable for postoperative surveillance, we examined the annual hazard rate of recurrence in a large multicenter set of patients with HCC detected by active surveillance, which is annotated as a standard surgical indication; and we compared the innate clinicopathologic risk factors related to recurrence in the early and late periods after resection in these patients. On the basis of findings based on these analyses, we intend eventually to propose an annualized hazard rate-based surveillance strategy for patients undergoing standard hepatectomy for HCC.

\section{Patients and Methods}

\section{The Multicenter Set of Patients}

A total of 2,604 consecutive patients aged $>19$ years who underwent primary hepatic resection for HCC between January 2007 and January 2015 in 3 university-based academic medical centers in South Korea (Asan Medical Center, Hanyang University Guri Hospital, and Kyung Hee University Hospital at Gangdong) were screened and considered for inclusion in this analysis. All the included patients had been under regular surveillance prior to HCC diagnosis. Surveillance activities were defined as performing abdominal ultrasound and measuring serum alpha-fetoprotein (AFP) twice or more at least 6 months apart for 18 months preceding the HCC diagnosis. None of the patients received preoperative neoadjuvant or bridging therapy before surgery or had severe renal or cerebro-cardiovascular disorders or uncontrolled metabolic disease, which might have led to death unrelated to the underlying liver disease. In order to include optimal candidates for surgical resection following the evidence-based practice guidelines [2, 13], patients were excluded if they had (1) Child-Turcott-Pugh class B or C liver function $(n=91)$; (2) any adjuvant chemotherapy after surgery $(n=90)$; (3) history of primary cancers other than HCC $(n=46)$; (4) multiple HCC nodules with at least one tumor $>5 \mathrm{~cm}$ across or tumors with gross vascular invasion (i.e., T3 or T4 tumors staged using the American Joint Committee on Cancer staging system based on pathologic findings; $n=359$ ) [14]; and (5) extrahepatic or lymphatic metastasis based on imaging findings $(n=100)$. In the end, a total of 1,918 patients with single HCCs of any size, or $>1$ tumor but none $>5 \mathrm{~cm}$ across (i.e., T1 or T2 tumors) that were curatively resected, and had well-preserved liver function, were eligible for this study (see online suppl. Fig. 1; for all online suppl. material, see www.karger.com/doi/10.1159/000518837). Curative resection was individually confirmed by postsurgical pathology, which was principally defined as a local radical procedure (R0) with tumor-negative resection margins without directly invaded adjacent organs. Ethical approval for our research protocol involving the study of human subjects was obtained from the institutional review board (IRB) at each participating hospital.

\section{Staging Work-Ups and Follow-Up after Surgery}

The evaluation and management processes employed by hepatologists and liver surgeons in Korea pre- and post-hepatectomy were as previously described $[15,16]$. Briefly, preoperative procedures included multiphase liver computed tomography (CT) and/ or magnetic resonance imaging (MRI), chest CT, and bone scans for staging intra- and extrahepatic tumor extension, in addition to detailed laboratory tests and serum AFP levels. After surgery, all patients were routinely followed-up with liver protocol dynamic CT or MRI scans covering most of the chest, as well as blood tests including AFP assay. These assessments were performed regularly at intervals of 6 months or less until a recurrent lesion appeared, or the patient was lost to follow-up. Apart from pathologic diagnosis, intrahepatic recurrences detected during follow-up were diagnosed as new nodules with the typical imaging hallmarks of HCC (i.e., arterial phase hyperenhancement with washout in the portal venous, delayed, or hepatobiliary phases), or newly detected or growing nodules with positive ancillary imaging features (i.e., mild-tomoderate T2 hyperintensity, restricted diffusion, hypointensity in the hepatobiliary phase, and interval growth generally indicating malignancy; and nonenhancing capsule, mosaic appearance, nodule-in-nodule architecture, and fat or blood products in the tumor particularly indicating HCC) [16]. During surveillance after resection, extrahepatic recurrence was identified by CT, MRI, and/or nuclear imaging studies, especially in cases with symptoms attributable to metastatic lesions, or with abnormal elevation of serum AFP without evidence of intrahepatic recurrence [16-18]. 


\section{Clinical Endpoints and Variables}

The primary endpoint for this study was the annualized hazard rate for the HCC recurrence of interest, with time to first recurrence (TTR) after surgery and overall survival following initial recurrence considered secondary endpoints. In order to examine risk factors for recurrence according to postoperative period, we used clinico-laboratory variables (etiology of liver disease, ChildTurcott-Pugh score, serum AFP level, and other diverse liver-related blood tests), and histological variables (fibrosis stage based on the METAVIR scoring system [19], size of tumor, tumor multiplicity, microscopic vascular and capsular invasion, and differentiation grade of tumor according to the 5th edition of the WHO classification [20]), together with age at diagnosis and gender.

\section{Statistical Analysis}

Annualized hazard rates of recurrence were estimated based on a piece-wise exponential survival model, which corresponded to the number of recurrences within a year divided by the total followup time during that year. To construct $95 \%$ confidence intervals of annualized hazard rates, we drew 500 bootstrap samples from the study sample; we repeated the estimation of annualized hazard rates on each of the bootstrap samples, and thus obtained the percentile bootstrap confidence interval from the 500 estimates. To visually depict how each baseline variable affected the recurrence hazard over time, we plotted smoothed hazard rates using a kernelbased method [21]. Bandwidth selection and boundary correction followed the methods proposed by Müller and Wang [22]. We estimated the distribution of hazard ratio estimates under the null hypothesis (hazard ratio $=1$ ) by shifting the empirical distribution of hazard ratios obtained from the 500 bootstrap samples so that the mean hazard ratio became 1 . Then to find the $p$ value of the hazard ratio, we calculated the probability of observing hazard ratios that were further from 1 than the hazard ratio we actually observed in the original samples, based on the estimated null distribution mentioned above. To make statistical inferences about hazard rates from the variables during years 4 and 5 , we used 500 bootstrap samples as we did for the annualized hazard rates. $\chi^{2}$ or Fisher's exact tests were used to examine relationships between categorical variables, and Student's $t$ test or Wilcoxon rank-sum test to compare mean values of continuous variables ( \pm standard deviations [SDs]). To identify baseline predictors of TTR in the early and late periods separated by the cut-off point (i.e., 5 years) identified from the chronological pattern of annualized hazard rates for postoperative events, we performed 2 separate Cox regression analyses. For the first, we built a multivariable Cox regression model, censoring all patients without recurrence within 5 years after surgery. In the second, we performed a landmark analysis that used 5 years after surgery as the landmark time-point. That is, we included only those patients who were recurrence-free for the first 5 postoperative years, thus excluding patients in whom recurrence events occurred during the first 5 years after surgery. For both models, we selected independent variables based on the $p$ values in the univariate analyses (i.e., $p<0.2$ ), considering multicollinearity between the variables, and model parsimony to avoid overfitting, in addition to clinical significance [23-27]. Overall survival time was defined as the period elapsed from the date of either time of initial recurrence to any-cause death, regardless of whether death was related to underlying liver disease. Mortality event-time distributions were analyzed using the Kaplan-Meier method with the log-rank test. Analyses were performed using R version 3.5.2 (Vienna, Austria).

Period-Specific Recurrence of Resected HCC
Table 1. Preoperative clinical and postoperative pathologic characteristics of the entire set of participants

\begin{tabular}{|c|c|}
\hline Variable & All patients $(n=1,918)$ \\
\hline \multicolumn{2}{|c|}{ Demographic and laboratory parameter } \\
\hline Male gender & $1,496(78.0)$ \\
\hline Age, years & $56.0 \pm 9.8$ \\
\hline \multicolumn{2}{|l|}{ Etiology of liver disease } \\
\hline HBV infection & $1,623(84.6)$ \\
\hline HCV infection & $104(5.4)$ \\
\hline Others & $191(10.0)$ \\
\hline Serum AST, IU/L & $49 \pm 38$ \\
\hline Serum ALT, IU/L & $42 \pm 37$ \\
\hline Platelet count, $\times 10^{3} / \mathrm{mm}^{3}$ & $163 \pm 57$ \\
\hline Prothrombin time, INR & $1.04 \pm 0.07$ \\
\hline Serum creatinine, $\mathrm{mg} / \mathrm{dL}$ & $0.9 \pm 0.5$ \\
\hline Serum albumin, $\mathrm{g} / \mathrm{dL}$ & $4.0 \pm 0.4$ \\
\hline Serum bilirubin, $\mathrm{mg} / \mathrm{dL}$ & $0.8 \pm 0.3$ \\
\hline Serum AFP level, ng/mL & $2,835.6 \pm 23,912.0$ \\
\hline Serum AFP $\geq 200 \mathrm{ng} / \mathrm{mL}$ & $533(27.8)$ \\
\hline \multicolumn{2}{|l|}{ Pathologic parameter } \\
\hline Maximal tumor diameter, $\mathrm{cm}$ & $4.0 \pm 2.8$ \\
\hline$<3$ & $827(43.1)$ \\
\hline $3-5$ & $713(37.2)$ \\
\hline$>5$ & $378(19.7)$ \\
\hline Tumor multiplicity & $174(9.1)$ \\
\hline Microscopic vascular invasion & $363(18.9)$ \\
\hline Capsular invasion & $138(7.1)$ \\
\hline \multicolumn{2}{|l|}{ Histological differentiation* } \\
\hline Well differentiated & 1,335 (69.6) \\
\hline Moderately differentiated & $560(29.2)$ \\
\hline Poorly differentiated & $23(1.2)$ \\
\hline \multicolumn{2}{|l|}{ Stage of liver fibrosis ${ }^{\dagger}$} \\
\hline F0 & $134(7.0)$ \\
\hline $\mathrm{F} 1$ & $329(17.2)$ \\
\hline $\mathrm{F} 2$ & $344(17.9)$ \\
\hline F3 & $55(2.9)$ \\
\hline $\mathrm{F} 4$ & $1,056(55.0)$ \\
\hline
\end{tabular}

Values are expressed as mean $\pm S D$, or frequency (\%). HBV, hepatitis $B$ virus; $H C V$, hepatitis C virus; AST, aspartate aminotransferase; ALT, alanine aminotransferase; INR, international normalized ratio; AFP, alpha-fetoprotein; SD, standard deviation. * Histological differentiation was based on $2019 \mathrm{WHO}$ classification. ${ }^{\dagger}$ Liver fibrosis was staged according to the METAVIR scoring system: F0, absence of fibrosis; F1, portal fibrosis without septa; F2, portal fibrosis with rare septa; F3, numerous septa without cirrhosis; F4, cirrhosis.

\section{Results}

\section{Characteristics of the Included Patients at the Time of Resection}

Table 1 summarizes the demographic, clinical, and pathological characteristics of the entire set of participants. Males predominated $(78.0 \%)$ with a mean age at 


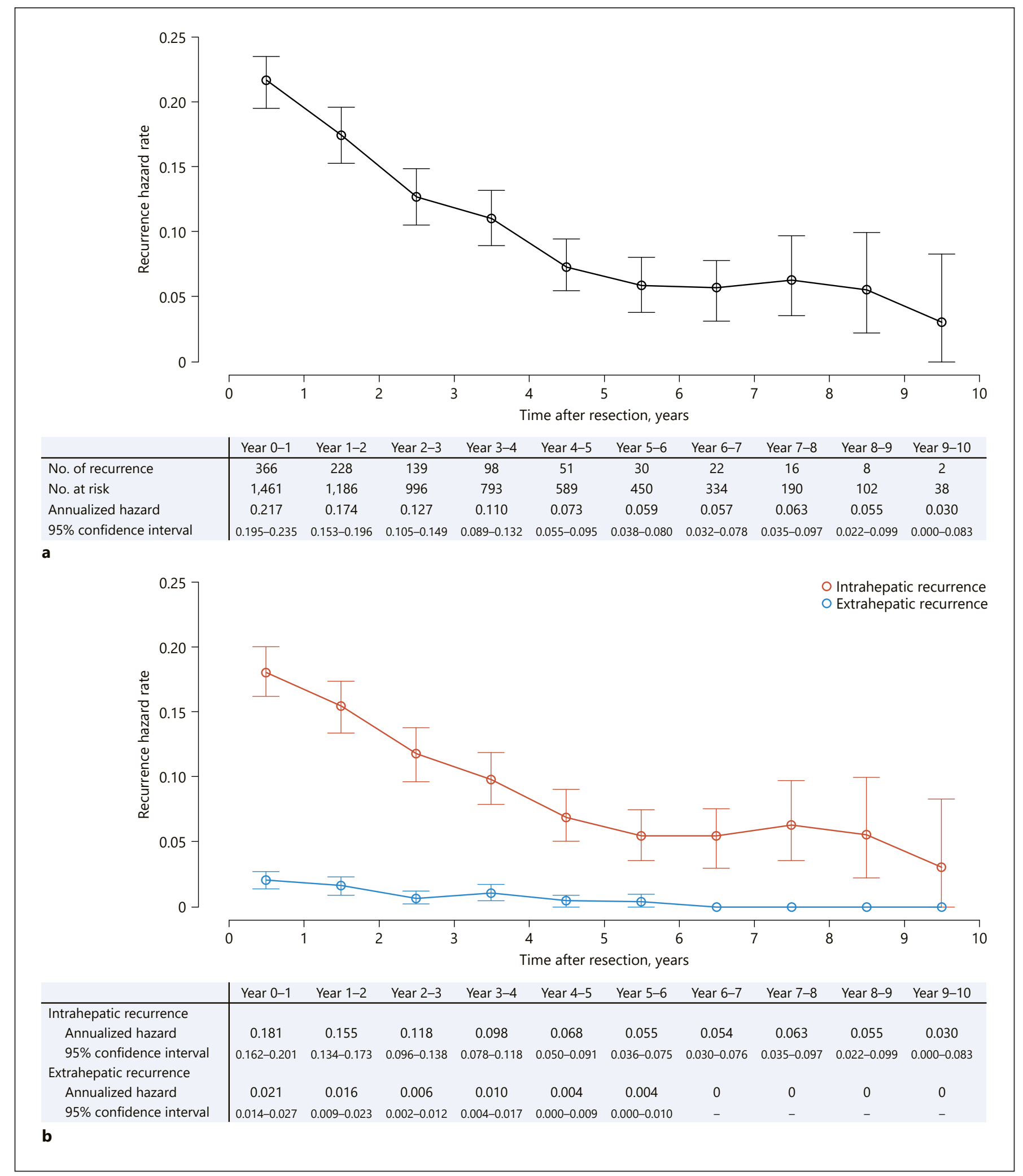

Fig. 1. Annual hazard rates of HCC recurrence after resection (a) for the entire cohort of 1,918 patients, and according to site of recurrence (intrahepatic or extrahepatic) (b). Vertical lines indicate $95 \%$ confidence intervals. HCC, hepatocellular carcinoma. 


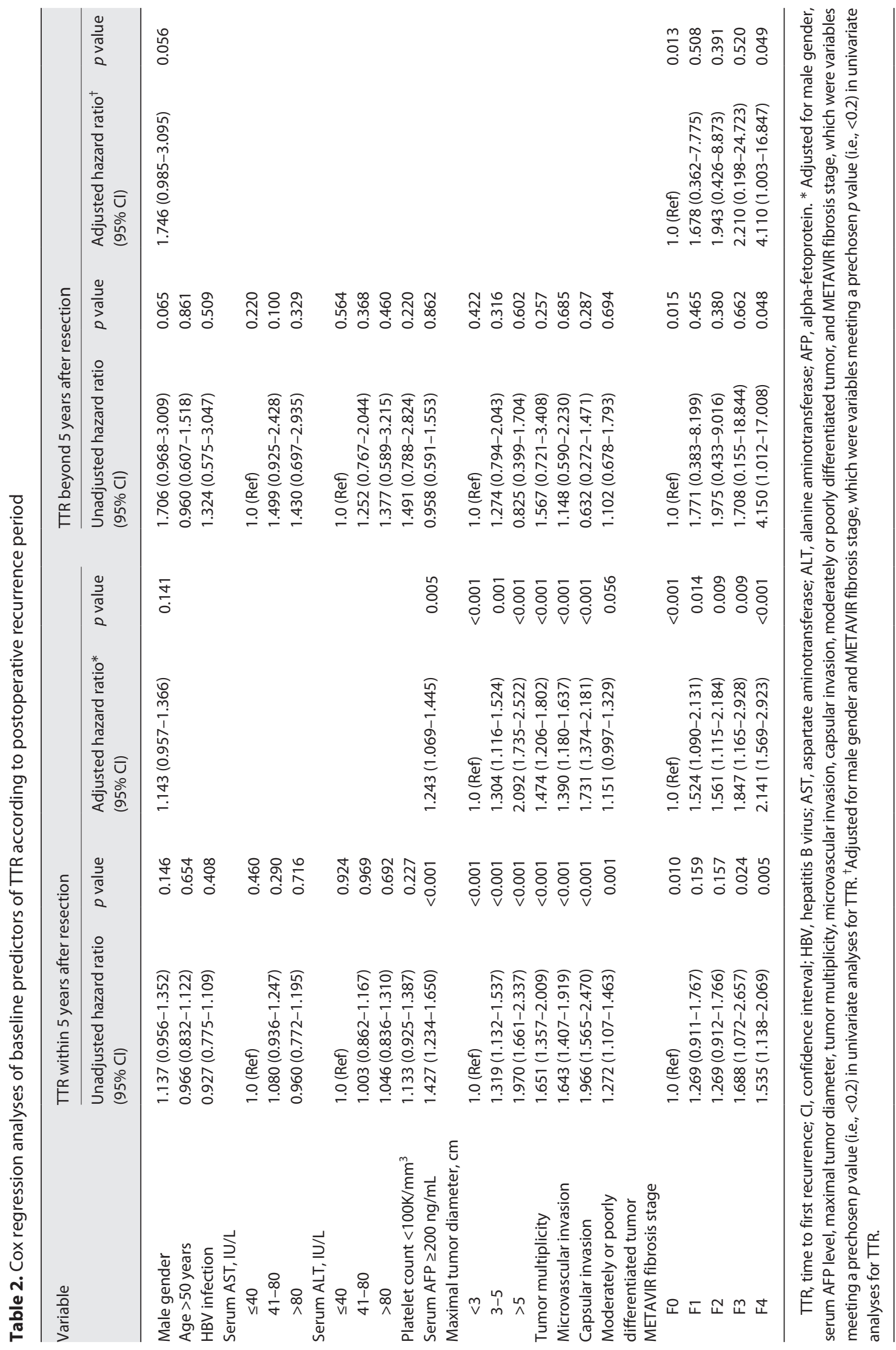


Table 3. Morphologic and spatial patterns of initial recurrence according to postoperative recurrence period

\begin{tabular}{llll}
\hline Variable & $\begin{array}{l}\text { Earlier relapse (within 5 years) } \\
(n=882)\end{array}$ & $\begin{array}{l}\text { Later relapse (beyond 5 years) } \\
(n=79)\end{array}$ & $p$ value \\
\hline Intrahepatic recurrence* $^{*}$ & $807(91.5)$ & $77(97.5)$ & 0.037 \\
$\quad$ Solitary tumor & $767(87.0)$ & $72(91.1)$ & 0.285 \\
$\quad$ Maximum diameter of tumor & $1.7 \pm 1.1 \mathrm{~cm}$ & $1.6 \pm 0.7 \mathrm{~cm}$ & 0.609 \\
$\quad$ Presence of PVTT & $12(1.4)$ & $0(0)$ & 0.355 \\
Extrahepatic recurrence* & $111(12.6)$ & $3(3.8)$ & 0.010 \\
$\quad$ Lung & $69(62.2)$ & $2(66.7)$ & - \\
$\quad$ Bone & $19(17.1)$ & $0(0)$ & - \\
$\quad$ Lymph node & $16(14.4)$ & $0(0)$ & - \\
$\quad$ Peritoneum & $12(10.8)$ & $1(33.3)$ & - \\
$\quad$ Adrenal gland & $5(4.5)$ & $0(0)$ & 0.020 \\
Serum AFP at recurrence & $531.9 \pm 3,608.4 \mathrm{ng} / \mathrm{mL}$ & $58.39 \pm 232.2 \mathrm{ng} / \mathrm{mL}$ & \\
\hline
\end{tabular}

Values are expressed as mean \pm SD, or frequency (\%). PVTT, portal vein tumor thrombosis; AFP, alpha-fetoprotein; SD, standard deviation. * 37 patients had both intrahepatic and extrahepatic lesions at initial recurrence. ${ }^{\dagger}$ Data on serum AFP levels at the time of initial recurrence were not available for 37 of the 961 patients with recurrences during the study period: 33 and 4 in the earlier and the later relapse groups, respectively.

hepatectomy of 56.0 years (SD, 9.8). Of the 1,918 patients, $84.6 \%$ were positive for hepatitis B virus (HBV), 27.8\% had serum AFP values $\geq 200 \mathrm{ng} / \mathrm{mL}$, and $9.1 \%$ had multiple tumors. Of 1,623 HBV-infected patients, 1,127 $(69.4 \%)$ received antiviral therapy at the time of hepatectomy. Based on the pathologic indices of the surgical specimens, about half of the patients (57.9\%) had METAVIR fibrosis scores of F3 (advanced fibrosis) or F4 (cirrhosis); the median size of the original tumors was $4.0 \mathrm{~cm}$ $(\mathrm{SD}, 2.8)$; and microvascular and capsular invasion of the tumors were detected by microscope in 363 (18.9\%) and $138(7.1 \%)$ patients, respectively; and $30.4 \%$ of the tumors were identified as being moderately or poorly differentiated according to the WHO classification [20].

\section{Changes over Time in Annual Hazards of Recurrence after Resection}

At a median follow-up time of 5.7 postoperative years (interquartile range, 3.6-7.9 years), HCCs had recurred in 961 (50.1\%) patients. Figure 1a depicts chronologically the hazard rates for overall recurrence at 1-year intervals following hepatectomy in the entire set of patients. The estimated hazard rate for recurrence peaked in the first postoperative year (21.7\%/year) and then gradually decreased until the fifth year (7.3\%/year). Thereafter, it remained stable at an annual rate below 6.5\% (range, 3.0$6.3 \% /$ year; and mean rate, $5.3 \% /$ year) until end-of-period censoring in year 10 . We note that the upper limit of annual HCC incidence in cirrhotic individuals due to any cause was previously estimated at approximately $8.0 \%$ [28-30]. While intrahepatic recurrences of HCC were the main contributors to this trend in hazards, the much lower hazard rates for extrahepatic recurrences persisted, especially after years $4-5$ (Fig. 1b). Similar findings were also observed in a subset of patients who had solitary HCCs ( $n=1,744$; online suppl. Fig. 2 ).

\section{Factors Contributing to the Period-Specific Recurrence Rates}

Overall recurrences during and after the first 5 years postresection were observed in 882 and 79 patients, respectively. Factors contributing to the cumulative recurrence rates in the 2 periods were estimated for the complete 1,918 series. Univariate and subsequent multivariable TTR analyses by postoperative period revealed that serum AFP $\geq 200 \mathrm{ng} / \mathrm{mL}$ (adjusted hazard ratio [95\% confidence interval], 1.243 [1.069-1.445]; $p=0.005)$, maximum size of tumor (1.304 [1.116-1.524] for 3-5 cm vs. $<3 \mathrm{~cm}$; and 2.092 [1.735-2.522] for $>5 \mathrm{~cm}$ vs. $<3 \mathrm{~cm} ; p=$ 0.001 and $p<0.001$, respectively), tumor multiplicity (1.474 [1.206-1.802]; $p<0.001)$, and pathologic parameters such as microvascular invasion (1.390 [1.180-1.637]; $p<0.001$ ), capsular invasion (1.731 [1.374-2.181]; $p<$ 0.001), METAVIR fibrosis stage (1.524 [1.090-2.131] for F1; 1.561 [1.115-2.184] for F2; 1.847 [1.165-2.928] for F3; and 2.141 [1.569-2.923] for F4, compared to F0; all Ps < 0.05 ) were significant predictors of recurrence during years $0-5$. Only cirrhosis (METAVIR F4) was significant- 
Table 4. Cox regression analysis of baseline predictors of time-to-extrahepatic recurrence after hepatectomy

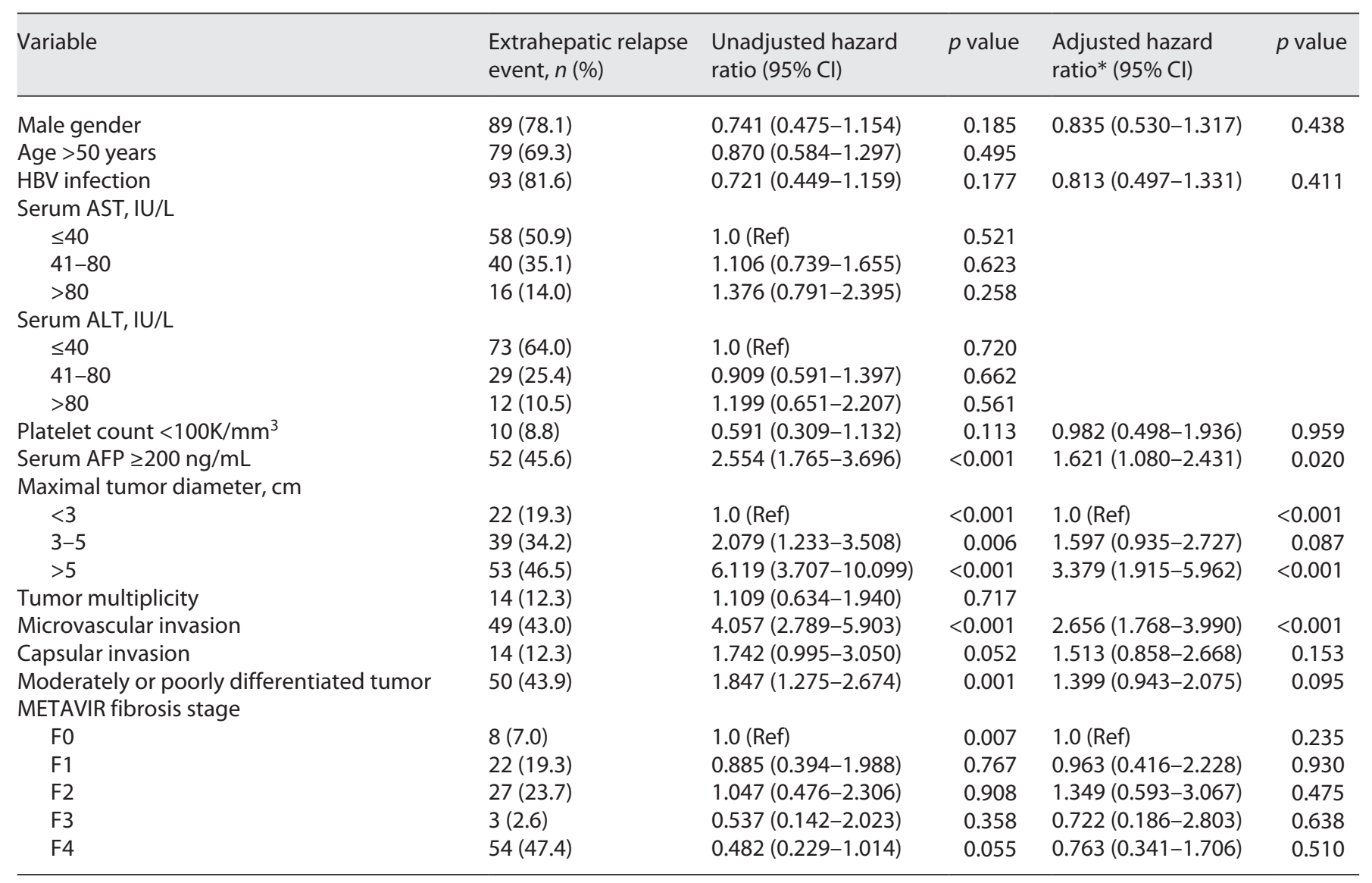

$\mathrm{Cl}$, confidence interval; HBV, hepatitis B virus; AST, aspartate aminotransferase; ALT, alanine aminotransferase; AFP, alpha-fetoprotein. * Adjusted for male gender, HBV infection, platelet count, serum AFP level, maximal tumor diameter, microvascular invasion, capsular invasion, moderately or poorly differentiated tumor, and METAVIR fibrosis stage, which were variables meeting a prechosen $p$ value (i.e., $<0.2)$ in univariate analyses for time-to-extrahepatic recurrence.

ly correlated with recurrence beyond 5 years, with an over-4-fold increase in risk relative to F0 (4.110 [1.00316.847]; $p=0.049$ ) (Table 2). When we investigated the difference in the pattern of recurrence hazards according to the presence of liver cirrhosis, we also observed hazard rates of $>8 \%$ after year 5 in cirrhotic patients, their rates during years 4 and 5 being significantly higher than in noncirrhotics ( 10.5 vs. $5.8 \%, p=0.004$; online suppl. Fig. 3).

\section{Clinical Patterns and Outcomes of Period-Specific \\ Recurrences}

First recurrences within and beyond 5 years postresection generally presented as new nodules in the liver (91.5 and $97.5 \%$, respectively; $p=0.037$; Table 3 ). Extrahepatic metastases at first recurrence, with or without intrahe- patic lesions, were detected in 111 patients $(12.6 \%)$ in years 0 and 5 compared with $3(3.8 \%)$ beyond year 5 ( $p=$ $0.010)$; the most common site was the lung $(62.3 \%)$ followed by bone (16.7\%). HCC recurrences in the earlier period had greater mean levels of serum AFP (531.9 \pm $3,608.4 \mathrm{ng} / \mathrm{mL}$ vs. $58.39 \pm 232.2 \mathrm{ng} / \mathrm{mL}, p=0.020)$. Significant independent baseline risk factors for disease outside the liver as initial recurrence were serum AFP $\geq 200$ $\mathrm{ng} / \mathrm{mL}$, maximal tumor size $>5 \mathrm{~cm}$, and microvascular invasion (hazard ratios [95\% confidence intervals], 1.621 [1.080-2.431]; 3.379 [1.915-5.962]; and 2.656 [1.7683.990], respectively; all $P_{\mathrm{s}}<0.05$, Table 4).

In terms of overall survival after initial recurrence at any site, the later recurrence group (years 5-10) had a better prognosis than their earlier counterparts (years 0-5) ( 89.4 vs. $75.0 \%$ and 64.5 vs. $60.7 \%$ at 3 and 5 years postre- 


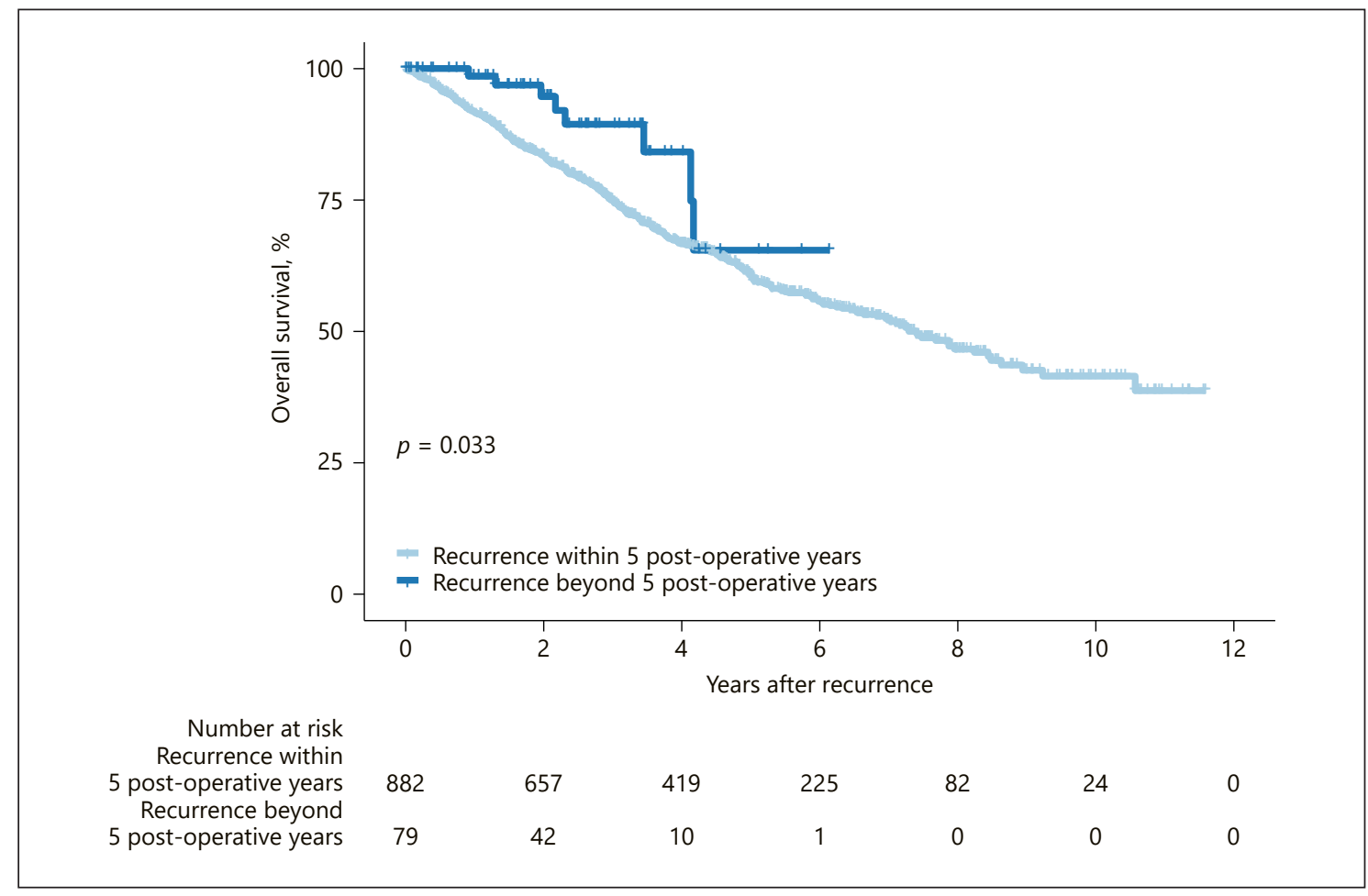

Fig. 2. Comparison of Kaplan-Meier survival curves in patients who experienced initial recurrence within less than, or after $>5$ years after hepatectomy for primary HCC. The late-period recurrence group had overall survival rates of 89.4 and $64.5 \%$ at 3 and 5 years after initial recurrence, respectively, significantly higher than those of the early period recurrence group (75.0 and $60.7 \%$ for the corresponding years; $p=0.033$ ). HCC, hepatocellular carcinoma.

currence, respectively, $p=0.033$; Fig. 2). In an additional Kaplan-Meier analysis, cumulative incidence rates for overall death at 3,5 , and 7 years after resection were 10.5 , 22.2 , and $32.1 \%$ for the subgroup with intrahepatic recurrence alone $(n=847) ; 31.9,42.6$, and $57.0 \%$ for extrahepatic recurrence alone $(n=77)$; and $43.3,69.3$, and $69.3 \%$ for both intra- and extrahepatic recurrences $(n=37)$, respectively $(P s<0.05$ for all between-group comparisons by log-rank test; online suppl. Fig. 4$)$.

\section{Discussion}

Ideally, postoperative surveillance programs in patients with any type of malignancy should be based on reliable estimates of the risk of tumor recurrence [12]. In this large-scale multicenter outcome study of optimal candidates for surgical resection for HCC according to the practice guidelines [13], we found that the peak hazard rate of natural recurrences involving mainly intrahe- patic disease was $21.7 \%$ during the 1st year of postoperative follow-up, followed by a steep decline in rate up to year 5 . In subsequent years, the hazard stabilized at an average yearly recurrence rate of $5.3 \%$ between the 5 th and 10th year postresection, which is comparable to that for noncancerous cirrhotic groups, and did not ever fall below $5 \%$ before the 9 th year [28-30]. A similar trend was noted for the most ideal surgical subseries of single HCCs, with a lower peak annual hazard rate of $18.1 \%$. However, HCC occurring on a background of cirrhosis had an ongoing recurrence risk exceeding the maximum annual incidence of HCC $(\approx 8 \%)$ in those surveilled cirrhotics who had no history of liver cancer but harbored the highest risk of developing HCC [28, 31].

Previous observational studies of resected HCCs reported 2 peaks of incidence of recurrences in the 1st year and the 4 th to 5 th year, respectively, after surgery. In contrast, no second peak was evident in our series, which was larger and had a longer follow-up [10,11]. Apart from the smaller sample sizes of the previous studies, the reason for 
this difference in recurrence pattern may have been that $>80 \%$ of the present cohort were HBV-infected, compared with $<40 \%$ of the previous cohorts, while $>50 \%$ of the latter harbored hepatitis C virus (HCV) $[10,11]$. An Italian randomized-controlled study found that despite an HCV clearance rate of only 7\%, chemopreventive interferon after HCC resection was able to half the late-recurrence peak [32]. Most of HCV-infected patients in the 2 earlier studies, many of whom were enrolled before the early 2000s when no effective anti-HCV agents were available, may not have been virologically cured, and this may have resulted in failure to prevent the second peak of recurrence $[10,32]$. In contrast, $\mathrm{HBV}$ was spontaneously inactivated or strongly suppressed by nucleot(s)ide analogues in most of our HBV-infected series, thanks to the fact that the national health insurance system in South Korea covers antiviral therapy for HCC patients with any level of viremia [33]. This finding should have reduced persistent hepatic necroinflammation and the related likelihood of late recurrence [34-36]. This explanation may support the reliability of our single-peak recurrence profile.

We found that the conventional risk factors such as high AFP level, tumor size, tumor multiplicity, and invasive microscopic features contributed to HCC recurrence in the first few years after resection, as reported previously $[7,10,37,38]$. Our observations and previous ones have shown that the condition of the surrounding liver is the only consistent predictive factor for recurrence within and beyond 5 years of surgery. These findings support the view that the later recurrences are mainly associated with de novo HCCs that may no longer have the characteristics of the initial tumors [2, 6, 10, 11, 37-39].

There is recent evidence that regular surveillance for recurrence improves the long-term survival of patients with resected HCC [38]. At this time, there is little direct information to guide the choice of optimal surveillance strategy after curative-intent surgery for HCC. The current recommendations are merely to surveil individuals more strictly for the first 1-2 years after HCC resection $[2,40]$. Although recurrences developing within 2 years usually have a poor prognosis, the choice of cut-off time was not driven by studies aimed at correctly scheduling postsurgical surveillance for HCC $[6,10,41]$. Since early detection of relapse surely provides the best chance of effective therapy for recurrent HCC, our chronological findings suggest that high-intensity surveillance after surgery would be preferable, particularly for the first 5 years when recurrence is more frequent and life-threatening, and should then revert to routine intensity (i.e., biannual) as in general cirrhotic patients. Prolonged close surveillance, together with special consideration for early liver transplantation, may be required for patients with liver cirrhosis, due to the sustained high risk of recurrence [42]. It is also important that $\mathrm{HBV} / \mathrm{HCV}$ infections be eliminated to minimize late recurrence for the duration of the patients' lifespans [10, 32, 43, 44].

Unfortunately, data on which to base an optimal modality of surveillance for recurrent HCC after resection remain scarce. We encountered a considerable number of extrahepatic recurrences during follow-up, mainly in the lungs: these lesions all developed in the first 5 years except for 3 events in later years, consistent with the trends in other surgical series $[45,46]$, and were associated with very poor survival. Therefore, early period surveillance should perhaps include pulmonary screening to detect these potentially disastrous lesions early. In addition, we suggest that patients with large initial tumors, elevated AFP, and/or microvascular invasion, who our data indicate are at higher risk of metastatic recurrence, should be preferentially enrolled in clinical trials of adjuvant therapy.

A limitation of this retrospective study is that although HBV infection did not correlate with recurrence outcomes (Table 2), it was present in the background liver in the majority of our multicenter series, unlike in Japanese and Western patients who mainly suffer from HCV disease - which is thought to be associated with higher rates of poorly differentiated tumors, vascular invasion, and cirrhosis, as well as to have a higher recurrence rate [47]. The results of our study, including the cut-off time of 5 years for postoperative surveillance, should be replicated in other clinical sets to determine if they can be generalized.

In conclusion, this long-term follow-up study provides updated insight into the chronological and morphological patterns of HCC as a function of the risks and timing of postresection recurrence. We present an evidence-based framework to help implement a period-dependent surveillance protocol in surgically treated patients with HCC. Further cost-effect studies should allow clinicians and surgeons to recommend a data-based frequency and intensity of screening for recurrent disease in resected patients.

\section{Statement of Ethics}

Ethical approval for our research protocol involving the study of human subjects was obtained from the IRB at each participating hospital (IRB No., 2019-0694 for Asan Medical Center, 2020-06- 
023 for Hanyang University Guri Hospital, and 2020-03-061 for Kyung Hee University Hospital at Gangdong), informed consent was waived given the retrospective nature of the study.

\section{Conflict of Interest Statement}

The authors declare no competing interests.

\section{Funding Sources}

This study was supported by grants from the Basic Science Research Program through the National Research Foundation of Korea funded by the Ministry of Science and ICT (NRF2017R1E1A1A01074298) and the research fund of Hanyang University (HY-201900000002619).

\section{Author Contributions}

H.I. Kim, J. An, and J.H. Shim contributed to the study concept and design, the acquisition, analysis and interpretation of data, drafting of the manuscript, and critical revision of the manuscript for important intellectual content. J.Y. Kim, H.P. Shin, G.-W. Song, and H.C. Lee contributed to the acquisition of data and critical revision of the manuscript for important intellectual content. S.Y. Park contributed to the statistical analysis and critical revision of the manuscript for important intellectual content.

\section{Data Availability Statement}

All data generated or analyzed during this study are included in this article and its online suppl. files. Further inquiries can be directed to the corresponding author.

\section{References}

1 Llovet JM, Schwartz M, Mazzaferro V. Resection and liver transplantation for hepatocellular carcinoma. Semin Liver Dis. 2005;25: 181.

2 European Association for the Study of the Liver. EASL clinical practice guidelines: management of hepatocellular carcinoma. J Hepatol. 2018 Jul;69(1):182-236.

3 Kumada T, Nakano S, Takeda I, Sugiyama K, Osada T, Kiriyama S, et al. Patterns of recurrence after initial treatment in patients with small hepatocellular carcinoma. Hepatology. 1997;25(1):87-92.

4 Takayama T, Makuuchi M, Hirohashi S, Sakamoto M, Yamamoto J, Shimada K, et al. Early hepatocellular carcinoma as an entity with a high rate of surgical cure. Hepatology. 1998;28:1241.

5 Poon RT, Fan ST, Ng IO, Lo CM, Liu CL, Wong J. Different risk factors and prognosis for early and late intrahepatic recurrence after resection of hepatocellular carcinoma. Cancer. 2000;89:500.

6 Portolani N, Coniglio A, Ghidoni S, Giovanelli M, Benetti A, Tiberio GA, et al. Early and late recurrence after liver resection for hepatocellular carcinoma: prognostic and therapeutic implications. Ann Surg. 2006 Feb; 243(2):229-35.

7 Chan AWH, Zhong J, Berhane S, Toyoda H, Cucchetti A, Shi K, et al. Development of pre and post-operative models to predict early recurrence of hepatocellular carcinoma after surgical resection. J Hepatol. 2018 Dec;69(6): 1284-93.

8 Hoshida Y, Villanueva A, Kobayashi M, Peix J, Chiang DY, Camargo A, et al. Gene expression in fixed tissues and outcome in hepatocellular carcinoma. N Engl J Med. 2008;359: 1995.
9 Chen YJ, Yeh SH, Chen JT, Wu CC, Hsu MT, Tsai SF, et al. Chromosomal changes and clonality relationship between primary and recurrent hepatocellular carcinoma. Gastroenterology. 2000 Aug;119(2):431-40.

10 Imamura H, Matsuyama Y, Tanaka E, Ohkubo T, Hasegawa K, Miyagawa S, et al. Risk factors contributing to early and late phase intrahepatic recurrence of hepatocellular carcinoma after hepatectomy. J Hepatol. 2003;38(2): 200-7.

11 Sasaki K, Shindoh J, Margonis GA, Nishioka Y, Andreatos N, Sekine A, et al. Effect of background liver cirrhosis on outcomes of hepatectomy for hepatocellular carcinoma. JAMA Surg. 2017 Mar 15;152(3):e165059.

12 Aalen OO, Gjessing HK. Understanding the shape of the hazard rate: a process point of view. Stat Sci. 2001;16(1):1-22.

13 Heimbach JK, Kulik LM, Finn RS, Sirlin CB, Abecassis MM, Roberts LR, et al. AASLD guidelines for the treatment of hepatocellular carcinoma. Hepatology. 2018 Jan;67(1):35880.

14 Amin ME, Greene SBFL. AJCC cancer staging manual. 8th ed. New York: Springer; 2017.

15 Lee SG, Hwang S. How I do it: assessment of hepatic functional reserve for indication of hepatic resection. J Hepatobiliary Pancreat Surg. 2005;12(1):38-43.

16 Korean Liver Cancer Association; National Cancer Center. 2018 Korean Liver Cancer Association-National Cancer Center Korea practice guidelines for the management of hepatocellular carcinoma. Gut Liver. 2019 May 15;13(3):227-99.

17 Katyal S, Oliver JH, Peterson MS, Ferris JV, Carr BS, Baron RL. Extrahepatic metastases of hepatocellular carcinoma. Radiology. 2000; 216:698.
18 Uchino K, Tateishi R, Shiina S, Kanda M, Masuzaki R, Kondo Y, et al. Hepatocellular carcinoma with extrahepatic metastasis: clinical features and prognostic factors. Cancer. 2011 Oct 1;117(19):4475-83.

19 Bedossa P, Poynard T. An algorithm for the grading of activity in chronic hepatitis $\mathrm{C}$. The METAVIR cooperative study group. Hepatology. 1996 Aug;24(2):289-93.

20 International Agency for Research on Cancer. WHO classification of tumours of the digestive system. 5th ed. Lyon: International Agency for Research on Cancer; 2019.

21 Hess KR, Serachitopol DM, Brown BW. Hazard function estimators: a simulation study. Stat Med. 1999 Nov 30;18(22):3075-88.

22 Müller HG, Wang JL. Hazard rates estimation under random censoring with varying kernels and bandwidths. Biometrics. 1994;50:61-76.

23 George M, Greenland S. Simulation study of confounder-selection strategies. Am J Epidemiol. 1993;138:923-36.

24 Harrell FE Jr. Regression modeling strategies: with applications to linear models, logistic and ordinal regression, and survival analysis. New York: Springer; 2016.

25 Harrell FE Jr, Lee KL, Mark DB. Multivariable prognostic models: issues in developing models, evaluating assumptions and adequacy, and measuring and reducing errors. Stat Med. 1996;15:361-87.

26 Steyerberg EW, Eijkemans MJ, Habbema JD. Stepwise selection in small data sets: a simulation study of bias in logistic regression analysis. J Clin Epidemiol. 1999;52:935-42.

27 Steyerberg EW, Eijkemans MJ, Harrell FE, Habbema JD. Prognostic modelling with logistic regression analysis: a comparison of selection and estimation methods in small data sets. Stat Med. 2000;19:1059-79. 
28 Herbst DA, Reddy KR. Risk factors for hepatocellular carcinoma. Clin Liver Dis. 2012 Dec;1(6):180-2.

29 Fattovich G, Stroffolini T, Zagni I, Donato F. Hepatocellular carcinoma in cirrhosis: incidence and risk factors. Gastroenterology. 2004 Nov;127(5 Suppl 1):S35-50.

30 Kanwal F, Singal AG. Surveillance for hepatocellular carcinoma: current best practice and future direction. Gastroenterology. 2019 Jul; 157(1):54-64.

31 Hanouneh IA, Alkhouri N, Singal AG. Hepatocellular carcinoma surveillance in the 21 st century: saving lives or causing harm? Clin Mol Hepatol. 2019 Sep;25(3):264-9.

32 Mazzaferro V, Romito R, Schiavo M, Mariani L, Camerini T, Bhoori S, et al. Prevention of hepatocellular carcinoma recurrence with alpha-interferon after liver resection in $\mathrm{HCV}$ cirrhosis. Hepatology. 2006 Dec;44(6):154354.

33 Cho EJ, Kim SE, Suk KT, An J, Jeong SW, Chung WJ, et al. Current status and strategies for hepatitis B control in Korea. Clin Mol Hepatol. 2017 Sep;23(3):205-11.

34 Chuma M, Hige S, Kamiyama T, Meguro T, Nagasaka A, Nakanishi K, et al. The influence of hepatitis B DNA level and antiviral therapy on recurrence after initial curative treatment in patients with hepatocellular carcinoma. J Gastroenterol. 2009;44(9):991-9.

35 Huang G, Lau WY, Wang ZG, Pan ZY, Yuan SX, Shen F, et al. Antiviral therapy improves postoperative survival in patients with hepatocellular carcinoma: a randomized controlled trial. Ann Surg. 2015 Jan;261(1):5666.
36 Wong GL, Tse YK, Chan HL, Yip TC, Tsoi $\mathrm{KK}$, Wong VW. Oral nucleos(t)ide analogues reduce recurrence and death in chronic hepatitis B-related hepatocellular carcinoma. Aliment Pharmacol Ther. 2016 Apr;43(7):80213.

37 Tabrizian P, Jibara G, Shrager B, Schwartz M, Roayaie S. Recurrence of hepatocellular cancer after resection: patterns, treatments, and prognosis. Ann Surg. 2015 May;261(5):94755.

38 Xu XF, Xing H, Han J, Li ZL, Lau WY, Zhou $\mathrm{YH}$, et al. Risk factors, patterns, and outcomes of late recurrence after liver resection for hepatocellular carcinoma: a multicenter study from China. JAMA Surg. 2019;154(3):20917.

39 Bilimoria MM, Lauwers GY, Doherty DA, Nagorney DM, Belghiti J, Do KA, et al. Underlying liver disease, not tumor factors, predicts long-term survival after resection of hepatocellular carcinoma. Arch Surg. 2001;136: 528-35.

40 Benson AB, D'Angelica MI, Abbott DE, Anaya DA, Anders R, Are C, et al. Hepatobiliary cancers, version 2.2021, NCCN clinical practice guidelines in oncology. J Natl Compr Canc Netw. 2021 May 1;19(5):541-65.

41 Poon RT. Differentiating early and late recurrences after resection of HCC in cirrhotic patients: implications on surveillance, prevention, and treatment strategies. Ann Surg Oncol. 2009 Apr;16(4):792-4.
42 Chan AC, Chan SC, Chok KS, Cheung TT, Chiu DW, Poon RT, et al. Treatment strategy for recurrent hepatocellular carcinoma: salvage transplantation, repeated resection, or radiofrequency ablation? Liver Transpl. 2013 Apr;19(4):411-9.

43 Hoshida Y. Risk of recurrence in hepatitis Brelated hepatocellular carcinoma: impact of viral load in late recurrence. J Hepatol. 2009 Nov;51(5):842-4.

44 Sasaki Y, Yamada T, Tanaka H, Ohigashi H, Eguchi $\mathrm{H}$, Yano M, et al. Risk of recurrence in a long-term follow-up after surgery in $417 \mathrm{pa}$ tients with hepatitis B- or hepatitis C-related hepatocellular carcinoma. Ann Surg. 2006 Nov;244(5):771-80.

45 Shimada K, Sano T, Sakamoto Y, Kosuge T. A long-term follow-up and management study of hepatocellular carcinoma patients surviving for 10 years or longer after curative hepatectomy. Cancer. 2005 Nov 1;104(9): 1939-47.

46 Zheng J, Kuk D, Gönen M, Balachandran VP, Kingham TP, Allen PJ, et al. Actual 10-year survivors after resection of hepatocellular carcinoma. Ann Surg Oncol. 2017 May;24(5): 1358-66.

47 Li Q, Li H, Qin Y, Wang PP, Hao X. Comparison of surgical outcomes for small hepatocellular carcinoma in patients with hepatitis $B$ versus hepatitis C: a Chinese experience. J Gastroenterol Hepatol. 2007 Nov;22(11): 1936-41. 